\title{
Validação de conteúdo de instrumento sobre a habilidade em sondagem nasogástrica
}

\section{Instrument content validation on nasogastric intubation skills}

Rosana Kelly da Silva Medeiros ${ }^{1}$, Marcos Antonio Ferreira Júnior ${ }^{2}$, Gilson de Vasconcelos Torres ${ }^{3}$, Allyne Fortes Vitor ${ }^{4}$, Viviane Euzébia Pereira Santos ${ }^{5}$, Elizabeth Barichello ${ }^{6}$

\footnotetext{
${ }^{1}$ Enfermeira, Mestre em Enfermagem. Enfermeira da Estatégia Saúde da Família da Prefeitura Municipal de São Gonçalo do Amarante. São Gonçalo do Amarante, RN, Brasil. E-mail: rosana_kelly@hotmail.com.

${ }^{2}$ Enfermeiro, Doutor em Saúde e Desenvolvimento na Região Centro-Oeste. Professor Adjunto da Universidade Federal do Rio Grande do Norte (UFRN). Natal, RN, Brasil. E-mail: marcos_nurse@hotmail.com.

${ }^{3}$ Enfermeiro, Doutor em Enfermagem Fundamental. Professor Titular da UFRN. Natal, Rio Grande do Norte, Brasil. E-mail: gvt@ufrnet.br.

${ }^{4}$ Enfermeira, Doutora em Enfermagem. Professora Adjunta da UFRN. Natal, Rio Grande do Norte, Brasil. E-mail: allynefortes@yahoo.com.br.

${ }^{5}$ Enfermeira, Doutora em Enfermagem. Professora Adjunta da UFRN. Natal, Rio Grande do Norte, Brasil. E-mail: vivianeepsantos@gmail.com.

${ }^{6}$ Enfermeira, Doutora em Enfermagem. Professora Adjunta da Universidade Federal do Triângulo Mineiro. Uberaba, MG, Brasil. E-mail: lizabaric@gmail.com.
}

\section{RESUMO}

Objetivou-se realizar a validação de conteúdo de um instrumento sobre a habilidade em sondagem nasogástrica com base no modelo de validação de conteúdo de Pasquali. Trata-se de estudo metodológico desenvolvido com 23 enfermeiros docentes de instituições públicas de ensino superior do Rio Grande do Norte, por meio da aplicação do Índice de Validação de Conteúdo (IVC) e do índice Kappa. Na avaliação geral, o instrumento obteve IVC com valores acima de 0,75, IVC total de 0,95 e Kappa total de 0,91. No entanto, algumas alterações foram realizadas e se concentraram nos requisitos sequência instrucional dos tópicos, clareza e vocabulário. Desse modo, o instrumento apresentou confiabilidade e fidedignidade para observar a qualidade da assistência de enfermagem e pode ser utilizado por serviços de saúde e instituições formadoras para avaliar a habilidade dos profissionais e alunos de graduação, no entanto exige outras fases para uma validação de conteúdo mais precisa e específica.

Descritores: Cuidados de Enfermagem; Intubação Gastrointestinal; Estudos de Validação.

\section{ABSTRACT}

The objective was to validate the content of an instrument on nasogastric intubation skills based on Pasquali's model of content validation. This was a methodological study conducted with 23 nursing teachers in public higher education institutions in Rio Grande do Norte. The Content Validation Index (CVI) and Kappa Index was applied. In the overall evaluation, the instrument obtained a CVI with values above 0.75 with a total CVI of 0.95 and a total Kappa of 0.91. However, some changes were made on the instructional sequence requirements of the topics, and to vocabulary. The instrument proved to be reliable and trustworthy in facilitating nursing care quality and can be used for health services and educational institutions to assess skill levels of professionals and undergraduate students. However, the instrument requires additional stages for a more precise and specific content validation.

Descriptors: Nursing Care; Intubation, Gastrointestinal; Validation Studies. 


\section{INTRODUÇÃO}

A sondagem nasogástrica é um procedimento comum para, dentre outras funções, suprir as necessidades nutricionais quando o paciente está impossibilitado de ingerir o alimento em quantidade suficiente para repor suas necessidades, mas é capaz de absorver nutrientes ${ }^{(1)}$.

Essa é a via de mais fácil acesso ao trato gastrointestinal, menor custo e a que mais se aproxima do processo fisiológico, pois permite a atuação das enzimas digestivas habituais, ideal para pacientes hospitalizados e criticamente doentes com função gastrointestinal preservada e sem grandes riscos de aspiração e refluxo gastroesofágico ${ }^{(2)}$.

A sondagem nasogástrica se constitui em uma técnica tradicional, que exige habilidade precedida de domínio do conhecimento para sua execução segura, visto que a realização desse procedimento pode provocar algumas complicações $^{(3)}$.

Dentre essas complicações, pode haver danos, por meio de traumas na inserção como perfuração esofágica e formação de pneumotórax, bem como inserção traqueal com o deslocamento da sonda para os pulmões e promoção de alimentação intrapulmonar, que pode resultar em pneumonia por aspiração e, consequente, óbito, além de lesões nasais e orofaríngeas, bem como estenose esofágica ${ }^{(4-6)}$.

A execução da técnica de sondagem nasogástrica se constitui em um procedimento complexo cada vez mais realizado por profissionais capacitados e legalmente habilitados como o enfermeiro ${ }^{(7)}$. No entanto, ainda há uma grande variação nessa prática ${ }^{(7)}$.

Nesse sentido, é essencial a capacitação permanente e formação adequada de enfermeiros, de modo a agir efetivamente nos procedimentos de sondagem nasogástrica e evitar complicações ao paciente ${ }^{(4)}$.

A apropriação desses conhecimentos necessita ser garantida na formação desses profissionais, uma vez que a identificação de fatores relacionados à realização inadequada do procedimento de sondagem nasogástrica fornece subsídios para a formação de profissionais mais capacitados e para o desenvolvimento e aprimoramento da assistência de enfermagem.

Diante disso, é necessário que por meio de instrumentos de medição válidos seja possível avaliar a qualidade da assistência de enfermagem ${ }^{(8)}$, ao passo que o reconhecimento da qualidade dos instrumentos tornase aspecto fundamental para a legitimidade e credibilidade dos resultados de uma pesquisa, o que reforça a importância do processo de validação(9).

Um estudo de validade de conteúdo pode fornecer informações sobre a representatividade e clareza de cada item de um instrumento, com a colaboração de especialistas que oferecem sugestões concretas para melhorar a medida ${ }^{(10)}$. A validade de conteúdo é um processo de julgamento que apresenta duas partes, a primeira constitui o desenvolvimento do instrumento e a segunda envolve a análise dos especialistas ${ }^{(10)}$.

O modelo de validação de conteúdo de Pasquali ${ }^{(11)}$, presente em pesquisas de enfermagem, envolve, principalmente a teoria da elaboração de instrumentos de medida de fenômenos subjetivos, com a composição de três conjuntos de procedimentos: teóricos, empíricos (experimentais) e analíticos (estatísticos).

Segundo os apontamentos de uma revisão ${ }^{(12)}$, os procedimentos envolvidos na inserção e posicionamento da sonda nasogástrica são incorporados na prática de enfermeiros sem estudos suficientes, especialmente estudos de validação. Desse modo, existe a necessidade de se avaliar criticamente as técnicas de inserção e confirmação do posicionamento da sonda, visto que a técnica de sondagem nasogástrica é comum nos serviços hospitalares de saúde e necessita ser mais bem fundamentada ${ }^{(12)}$.

Assim, diante das responsabilidades exigidas na execução do procedimento, os riscos associados e a importância de uma assistência baseada em conhecimentos, o objetivo desse estudo é realizar a validação de conteúdo de um instrumento sobre a 
habilidade em sondagem nasogástrica com base no modelo de validação de conteúdo de Pasquali ${ }^{(11)}$.

\section{MÉTODO}

Estudo metodológico, com abordagem quantitativa de tratamento e análise de dados, realizado no período de junho a setembro de 2012 após a obtenção do parecer favorável do Comitê de Ética em Pesquisa do Hospital Universitário Onofre Lopes da Universidade Federal do Rio Grande do Norte sob CAAE no 0002.0.294.000-10.

O estudo foi desenvolvido em três etapas, na primeira (A) a construção do instrumento um roteiro de observação estruturado, do tipo checklist composto por 28 itens, divididos em três blocos (observações iniciais, técnica de sondagem nasogástrica e observações finais), que corresponderam aos passos para se observar a habilidade técnica acerca da sondagem nasogástrica, por meio de recomendações atualizadas na literatura. $\mathrm{Na}$ segunda etapa (B), procedeu-se à identificação, seleção e convite dos especialistas a fim de realizar o julgamento dos itens do instrumento; e a terceira etapa (C) correspondeu à validação de conteúdo dos instrumentos com a verificação do nível de concordância entre os juízes.

A Resolução da Diretoria Colegiada (RDC) nํ 63, de 6 de julho de 2000 que aprova o regulamento técnico para os requisitos mínimos exigidos à Terapia de Nutrição Enteral $^{(13)}$; a Resolução do Conselho Federal de Enfermagem no 277 de $2003^{(14)}$ e artigos científicos ${ }^{(2,15-16)}$, constituíram os principais referenciais teóricos para a construção do instrumento.

A amostra foi selecionada por intencionalidade com inclusão de docentes da disciplina de Semiologia e/ou Semiotécnica de Enfermagem, com no mínimo um ano de experiência na disciplina e atuação nas referidas universidades públicas do Rio Grande do Norte.

A segunda etapa foi realizada por meio de um contato com as coordenações dos cursos de graduação em Enfermagem da Universidade Federal do Rio Grande do Norte (UFRN) - campus central e campus Santa Cruz - e
Universidade do Estado do Rio Grande do Norte (UERN) campus Mossoró e campus Caicó, para solicitação dos contatos (e-mail e telefone) do corpo docente da disciplina de Semiologia e/ou Semiotécnica de Enfermagem para envio de uma carta convite por correio eletrônico, com os objetivos do estudo e a justificativa do processo de validação.

Dessa forma, 30 docentes foram convidados, dos quais 24 se disponibilizaram a participar da pesquisa, um foi excluído por não completar adequadamente o instrumento de coleta de dados, o que resultou em um total de 23 docentes. O Termo de Consentimento Livre e Esclarecido (TCLE) e o instrumento foram entregues pessoalmente aos docentes, e estabelecido o prazo de 15 dias para a devolução do material analisado e com o parecer final.

Os docentes selecionados para validação de conteúdo constituíram um total de 23 juízes representados de acordo com a instituição de atuação na Tabela 1. 
Tabela 1: Caracterização dos juízes participantes da pesquisa por instituição de atuação. Natal, RN, Brasil, 2013.

\begin{tabular}{|c|c|c|c|c|c|c|}
\hline \multirow{3}{*}{ Caracterização } & \multicolumn{4}{|c|}{ Instituição de Atuação } & \multirow{2}{*}{\multicolumn{2}{|c|}{ Total }} \\
\hline & \multicolumn{2}{|c|}{ UFRN } & \multicolumn{2}{|c|}{ UERN } & & \\
\hline & $n$ & $\%$ & $\mathrm{n}$ & $\%$ & $n$ & $\%$ \\
\hline \multicolumn{7}{|l|}{ Sexo } \\
\hline Masculino & 1 & 4,3 & 2 & 8,7 & 3 & 13 \\
\hline Feminino & 16 & 69,6 & 4 & 17,4 & 20 & 87 \\
\hline \multicolumn{7}{|l|}{ Outra atividade na enfermagem } \\
\hline Assistencial & 4 & 17,4 & 1 & 4,3 & 5 & 21,7 \\
\hline Gerencial & - & - & 1 & 4,3 & 1 & 4,3 \\
\hline Ambas & 1 & 4,3 & - & - & 1 & 4,3 \\
\hline Não & 12 & 52,2 & 4 & 17,4 & 16 & 69,6 \\
\hline \multicolumn{7}{|l|}{ Qualificação profissional } \\
\hline Doutorado & 3 & 13 & - & - & 3 & 13 \\
\hline Mestrado acadêmico & 13 & 56,5 & 5 & 21,7 & 18 & 78,3 \\
\hline Especialização & 1 & 4,3 & 1 & 4,3 & 2 & 8,7 \\
\hline \multicolumn{7}{|l|}{ Tempo de experiência na docência } \\
\hline 1 a 5 anos & 10 & 43,5 & 4 & 17,4 & 14 & 60,9 \\
\hline 6 a 10 anos & 3 & 13 & 2 & 8,7 & 5 & 21,7 \\
\hline$>10$ anos & 4 & 17,4 & - & - & 4 & 17,4 \\
\hline \multicolumn{7}{|c|}{ Tempo de experiência em semiologia e/ou semiotécnica de enfermagem } \\
\hline 1 a 5 anos & 13 & 56,5 & 5 & 21,7 & 18 & 78,3 \\
\hline 6 a 10 anos & 2 & 8,7 & 1 & 4,3 & 3 & 13 \\
\hline$>10$ anos & 2 & 8,7 & - & - & 2 & 8,7 \\
\hline
\end{tabular}

Os juízes avaliaram cada um dos 28 itens e os classificaram como adequado, adequado com alterações ou inadequado. Por meio de códigos numéricos, explicaram os motivos de alteração ou inadequação de acordo com requisitos adaptados dos critérios sugeridos por Pasquali ${ }^{(11)}$ : utilidade/pertinência (1), consistência (2), clareza (3), objetividade (4), simplicidade (5), exequível (6), atualização (7), vocabulário (8), precisão (9), sequência instrucional de tópicos (10). A avaliação global do instrumento também foi realizada com relação aos requisitos supracitados.

Após a avaliação dos instrumentos, procedeu-se a validação de conteúdo com aplicação do Índice de Validade de Conteúdo (IVC) e do Índice Kappa (K) para verificação do nível de concordância e nível de consistência (fidedignidade) dos juízes em relação à permanência ou não dos itens dos instrumentos.

Os valores de Kappa variam de -1 a +1 , quanto mais próximo de um, melhor o nível de concordância entre os observadores. Sua distribuição e os respectivos níveis de interpretação são: $<0,00=$ ruim; 0,00 a 0,20= fraco; 0,21 a $0,40=$ sofrível; 0,41 a $0,60=$ regular; 0,61 a $0,80=$ bom; 0,81 a 0,99= ótimo; $1,00=$ perfeito. Como critério de aceitação neste estudo, foi estabelecido a concordância superior a 0,61 entre os juízes.

O Índice de Validade de Conteúdo (IVC) mede a proporção ou porcentagem de juízes que estão em concordância sobre determinados aspectos do instrumento e de seus itens ${ }^{(10)}$. Para se avaliar todo instrumento, a forma utilizada neste estudo foi a média dos valores dos itens calculados separadamente, quando foram somados todos os IVC calculados separadamente e divididos pelo número de itens do instrumento.

A fim de estipular a taxa de concordância aceitável entre os juízes foram estabelecidos os valores recomendados de no mínimo 0,75 entre eles para servirem de critério de decisão sobre a pertinência e/ou aceitação do item ${ }^{(10-11)}$.

Os dados foram tabulados no programa Microsoft Excel 2010 e analisados a partir de leitura reflexiva e estatística descritiva. Ao fim da análise dos dados, os 
instrumentos foram reformulados de acordo com as orientações e sugestões dos juízes.

\section{RESULTADOS}

A Tabela 2 representa o julgamento em relação aos itens que compõem o instrumento de habilidade em sondagem nasogástrica e demonstra que nenhum deles foi avaliado como inadequado e todos obtiveram concordância dentro do nível estabelecido (IVC > 0,75 e Kappa> 0,61).

Tabela 2: Julgamento entre os juízes sobre itens do instrumento acerca da técnica de sondagem nasogástrica. Natal, RN, Brasil, 2013.

\begin{tabular}{|c|c|c|c|c|c|c|}
\hline \multirow{3}{*}{ Etapas } & \multicolumn{6}{|c|}{ Julgamento } \\
\hline & \multicolumn{2}{|c|}{ Adequado } & \multicolumn{2}{|c|}{$\begin{array}{l}\text { Adequado c/ } \\
\text { alterações }\end{array}$} & \multirow[t]{2}{*}{ IVC } & \multirow[t]{2}{*}{ Kappa } \\
\hline & $\mathbf{n}$ & $\%$ & $\mathbf{n}$ & $\%$ & & \\
\hline \multicolumn{7}{|l|}{ Observações iniciais } \\
\hline Verifica dados no prontuário do usuário & 22 & 95,7 & 1 & 4,4 & 0,96 & 0,91 \\
\hline Higieniza as mãos & 23 & 100,0 & - & - & 1,00 & 1,00 \\
\hline Seleciona o material e os instrumentos necessários & 19 & 82,6 & 4 & 17,4 & 0,83 & 0,70 \\
\hline Identifica-se para o paciente e explica o procedimento a ser realizado & 22 & 95,7 & 1 & 4,4 & 0,96 & 0,91 \\
\hline \multicolumn{7}{|l|}{ Técnica de sondagem nasogástrica } \\
\hline Calça luvas & 18 & 78,3 & 5 & 21,7 & 0,78 & 0,64 \\
\hline Coloca o paciente em posição de Fowler & 22 & 95,7 & 1 & 4,4 & 0,96 & 0,91 \\
\hline Examina a sonda & 21 & 91,3 & 2 & 8,7 & 0,91 & 0,83 \\
\hline Protege o tórax do paciente & 23 & 100,0 & - & - & 1,00 & 1,00 \\
\hline Examina as fossas nasais e seleciona a mais permeável & 22 & 95,7 & 1 & 4,4 & 0,96 & 0,91 \\
\hline $\begin{array}{l}\text { Determina o comprimento da sonda a ser introduzido pela medição } \\
\text { NOX }\end{array}$ & 21 & 91,3 & 2 & 8,7 & 0,91 & 0,83 \\
\hline Marca a medida da distância determinada & 21 & 91,3 & 2 & 8,7 & 0,91 & 0,83 \\
\hline Posiciona o paciente & 19 & 82,6 & 4 & 17,4 & 0,83 & 0,70 \\
\hline Lubrifica a sonda com anestésico & 21 & 91,3 & 2 & 8,7 & 0,91 & 0,83 \\
\hline Lubrifica a narina do paciente com anestésico & 20 & 87,0 & 3 & 13,0 & 0,87 & 0,76 \\
\hline Introduz a sonda delicadamente na narina & 23 & 100,0 & - & - & 1,00 & 1,00 \\
\hline Em caso de resistência, pára e retrocede & 22 & 95,7 & 1 & 4,3 & 0,96 & 0,91 \\
\hline Em caso de persistência na resistência, retira a sonda & 23 & 100,0 & - & - & 1,00 & 1,00 \\
\hline $\begin{array}{l}\text { Observa sinais de tosse, dificuldade respiratória, cianose ou agitação, } \\
\text { retirando e reintroduzindo }\end{array}$ & 22 & 95,7 & 1 & 4,3 & 0,96 & 0,91 \\
\hline Introduz a sonda lentamente até o mensurado & 23 & 100,0 & - & - & 1,00 & 1,00 \\
\hline Confirma a localização da sonda com testes de posicionamentos & 20 & 87,0 & 3 & 13,0 & 0,87 & 0,76 \\
\hline Fecha a conexão da sonda & 23 & 100,0 & - & - & 1,00 & 1,00 \\
\hline Em caso de drenagem, conecta a sonda ao coletor & 23 & 100,0 & - & - & 1,00 & 1,00 \\
\hline Retira as luvas & 23 & 100,0 & - & - & 1,00 & 1,00 \\
\hline Fixa a sonda & 23 & 100,0 & - & - & 1,00 & 1,00 \\
\hline \multicolumn{7}{|l|}{ Observações finais } \\
\hline Deixa o paciente em posição confortável & 23 & 100,0 & - & - & 1,00 & 1,00 \\
\hline Organiza o ambiente & 23 & 100,0 & - & - & 1,00 & 1,00 \\
\hline Higieniza as mãos & 23 & 100,0 & - & - & 1,00 & 1,00 \\
\hline Registra o procedimento no prontuário & 23 & 100,0 & - & - & 1,00 & 1,00 \\
\hline
\end{tabular}

Legenda: NOX - Nariz-Orelha-Xifoide

Dentre os 28 itens do instrumento, 13 apresentaram índice de concordância perfeito (Kappa $=1,00) ; 10$ apresentaram nível de concordância ótimo (Kappa de 0,81 a 0,99) cinco apresentaram nível de concordância bom (Kappa de 0,61 a 0,80), com Kappa total entre os itens do instrumento de 0,87 . Em relação ao IVC, todos os itens atingiram níveis acima de 0,75 e se obteve IVC total do instrumento de 0,95 . 
Foram 15 itens considerados adequados com alterações, dos quais três pertenciam ao bloco das observações iniciais e 12 ao da técnica de sondagem nasogástrica. No Quadro 1, são apresentadas as sugestões realizadas pelos juízes para que os itens considerados adequados com alterações sejam reformulados e melhorados.

No parecer final dos juízes acerca do instrumento, baseado nos 10 requisitos de avaliação, todos os requisitos adaptados dos critérios sugeridos por Pasquali obtiveram índice Kappa bom e IVC acima de 0,75, como mostra a Tabela 3. Desses, utilidade/pertinência, simplicidade e exequível receberam escore máximo de concordância. Em contrapartida, o item sequência instrucional dos tópicos apresentou índice menor em relação aos demais requisitos. Assim, esse requisito e outros que não apresentaram escore máximo de concordância, bem como os 15 itens considerados adequados com alterações foram analisados para adaptação, a fim de viabilizar a construção da versão final do instrumento.

Na avaliação geral, o instrumento da habilidade sobre técnica de sondagem nasogástrica obteve IVC com valores acima de 0,75 , bem como apresentação de IVC total de 0,95 e Kappa total de 0,91, o que demonstra a alta confiabilidade e fidedignidade do instrumento para observar a qualidade da assistência de enfermagem.

Tabela 3: Parecer final dos juízes acerca do instrumentoda técnica de sondagem nasogástrica. Natal, RN, Brasil, 2013.

\begin{tabular}{|c|c|c|c|c|c|c|}
\hline \multirow{3}{*}{ Requisitos } & \multicolumn{6}{|c|}{ Parecer final } \\
\hline & \multicolumn{2}{|c|}{ Adequado } & \multicolumn{2}{|c|}{ Adequado c/ alterações } & \multirow{2}{*}{ IVC } & \multirow{2}{*}{ Kappa } \\
\hline & $n$ & $\%$ & $n$ & $\%$ & & \\
\hline Utilidade/ pertinência & 23 & 100,0 & - & - & 1,00 & 1,00 \\
\hline Consistência & 22 & 95,7 & 1 & 4,3 & 0,96 & 0,91 \\
\hline Clareza & 22 & 95,7 & 1 & 4,3 & 0,96 & 0,91 \\
\hline Objetividade & 22 & 95,7 & 1 & 4,3 & 0,96 & 0,91 \\
\hline Simplicidade & 23 & 100,0 & - & - & 1,00 & 1,00 \\
\hline Exequível & 23 & 100,0 & - & - & 1,00 & 1,00 \\
\hline Atualização & 22 & 95,7 & 1 & 4,3 & 0,96 & 0,91 \\
\hline Vocabulário & 22 & 95,7 & 1 & 4,3 & 0,96 & 0,91 \\
\hline Precisão & 22 & 95,7 & 1 & 4,3 & 0,96 & 0,91 \\
\hline Sequência instrucional dos tópicos & 18 & 78,3 & 5 & 21,7 & 0,78 & 0,64 \\
\hline
\end{tabular}


Quadro 1: Sugestões dos juízes acerca dos itens considerados adequados com alterações. Natal, RN, Brasil, 2013.

\begin{tabular}{|c|c|c|c|}
\hline Bloco & Itens & Requisitos avaliados & Sugestões dos juízes \\
\hline \multirow{6}{*}{ 1. Observações Iniciais } & 1.1. Verifica dados no prontuário do usuário & Clareza & Detalhar melhor a etapa no instrumento. \\
\hline & \multirow{4}{*}{ 1.3. Seleciona o material e os instrumentos necessários } & Consistência; Vocabulário; & Substituir o verbo "seleciona" por "reúne". \\
\hline & & Atualização; & Suprimir a palavra "instrumentos". \\
\hline & & Precisão. & Enumerar os materiais necessários. \\
\hline & & - & Observar na literatura o uso de luvas e gazes estéreis. \\
\hline & $\begin{array}{l}\text { 1.4. Identifica-se para o paciente e explica o procedimento } \\
\text { a ser realizado }\end{array}$ & Sequência instrucional dos tópicos & Colocar como item 1.2 \\
\hline \multirow{19}{*}{$\begin{array}{l}\text { 2. Técnica de Sondagem } \\
\text { Nasogástrica }\end{array}$} & \multirow{3}{*}{ 2.1. Calça luvas } & Clareza; & $\begin{array}{l}\text { Especificar o tipo de luva (estéril ou não estéril). Colocar } \\
\text { após item 2.3. }\end{array}$ \\
\hline & & Atualização; & Colocar após item 2.5. \\
\hline & & Sequência instrucional dos tópicos. & - \\
\hline & 2.2. Coloca o paciente em posição de Fowler & Sequência instrucional dos tópicos & Colocar como item 2.1. \\
\hline & \multirow{2}{*}{ 2.3. Examina a sonda } & \multirow{2}{*}{$\begin{array}{l}\text { Objetividade; Vocabulário; Sequência } \\
\text { instrucional dos tópicos. }\end{array}$} & Contemplado no item 1.3. \\
\hline & & & Detalhar melhor a etapa no instrumento. \\
\hline & 2.5. Examina as fossas nasais e seleciona a mais permeável & Precisão & Detalhar sobre calibre da sonda no item. \\
\hline & $\begin{array}{l}\text { 2.6 Determina o comprimento da sonda a ser introduzido } \\
\text { pela medição NOX }\end{array}$ & Clareza; Vocabulário. & Observar na literatura o uso da sigla NOX. \\
\hline & 2.7. Marca a medida da distância determinada & Clareza & $\begin{array}{l}\text { Reformular a redação do item para: marca a sonda no } \\
\text { comprimento determinado anteriormente. }\end{array}$ \\
\hline & \multirow{4}{*}{ 2.8. Posiciona o paciente } & \multirow{4}{*}{$\begin{array}{l}\text { Clareza; Sequência instrucional dos } \\
\text { tópicos }\end{array}$} & $\begin{array}{l}\text { Deixar claro que é o posicionamento da cabeça do } \\
\text { paciente. }\end{array}$ \\
\hline & & & Contemplado no item 2.2 . \\
\hline & & & Colocar antes do item 2.7. \\
\hline & & & Contemplado no item 2.11. \\
\hline & 2.9. Lubrifica a sonda com anestésico & Sequência instrucional dos tópicos & Colocar após o item 2.10. \\
\hline & \multirow{2}{*}{ 2.10. Lubrifica a narina do paciente com anestésico } & \multirow{2}{*}{$\begin{array}{l}\text { Simplicidade; Sequência instrucional } \\
\text { dos tópicos }\end{array}$} & Detalhar melhor a etapa no instrumento. \\
\hline & & & Colocar antes do item 2.9. \\
\hline & 2.12. Em caso de resistência, para e retrocede & Clareza & $\begin{array}{l}\text { Deixar claro que após retroceder a sonda, esta é } \\
\text { reintroduzida. }\end{array}$ \\
\hline & $\begin{array}{l}\text { 2.14. Observa sinais de tosse, dificuldade respiratória, } \\
\text { cianose ou agitação, retirando e reintroduzindo }\end{array}$ & Clareza & Reformular a redação do item. \\
\hline & $\begin{array}{l}\text { 2.16. Confirma a localização da sonda com testes de } \\
\text { posicionamentos }\end{array}$ & Consistência; Clareza. & Detalhar os testes de posicionamento. \\
\hline
\end{tabular}




\section{DISCUSSÃO}

Com base no julgamento dos juízes para validação do instrumento, no bloco das observações iniciais foram apontadas sugestões para o melhoramento dos itens 1.1, 1.3 e 1.4. Em relação ao item 1.1 "verifica dados no prontuário do usuário", este item se refere à verificação dos dados de identificação do usuário, do diagnóstico e dados referente à nutrição enteral, caso a indicação da sonda seja para viabilizar alimentação enteral, como recomendado pela $\mathrm{RDC}^{(13)}$.

O item 1.3 "seleciona o material e os instrumentos necessários", foi modificado para "reúne os materiais necessários", conforme sugestões dos juízes. No entanto, as recomendações quanto à especificação do material não foram atendidas por se tratar de um instrumento conciso do tipo checklist para observação da prática de enfermagem durante a técnica de inserção de sonda nasogástrica.

Diante das sugestões dos juízes, vale ressaltar que alguns estudos ${ }^{(17-18)}$ indicam 0 uso de luvas de procedimento e gazes limpas, bem como apontam os materiais descritos no instrumento para adequação do item, tais como sonda prescrita, cuba rim ou bandeja, gaze limpa, luvas de procedimento, esparadrapo/micropore, anestésico gel hidrossolúvel tópico, seringa de $20 \mathrm{ml}$, estetoscópio, toalha ou papel toalha, além de coletor aberto e dispositivo intermediário de látex ou silicone que dependem da finalidade do procedimento.

É importante destacar que o uso de gel hidrossolúvel previne a pneumonia aspirativa por óleo, caso a sonda acidentalmente entre na traqueia, no entanto seu uso reduz o reflexo do engasgo, devido ao efeito anestésico da lidocaína na faringe, o que promove a redução de taxa de sucesso de posicionamento da sonda com aumento de inserção traqueal ${ }^{(19)}$.

O uso do estetoscópio, a fim de verificar os ruídos hidroaéreos antes do procedimento e a instilação de ar para confirmar posicionamento da sonda no estômago, tratado mais à frente, é contestado por alguns estudos, visto que ruídos intestinais e flatos ausentes permitem a nutrição enteral, uma vez que os ruídos são em decorrência da movimentação de ar no intestino e, muitas vezes, estão ausentes ou fracos, mesmo quando o intestino funciona normalmente, além disso o método auscultatório é impreciso na diferenciação entre a inserção traqueal ou gástrica da sonda ${ }^{(16,20)}$. Desse modo o estetoscópio foi eliminado dos instrumentos necessários à técnica de sondagem nasogástrica.

A sequência instrucional do tópico 1.4 "identifica-se para o paciente e explica o procedimento a ser realizado" foi alterada de acordo com a recomendação, e passa a corresponder ao item 1.2. A comunicação com o paciente é essencial para o cuidado, pois é importante que o profissional de enfermagem enfatize os aspectos positivos que propiciam a adesão ao tratamento, com enfoque no uso da sonda como uma alternativa viável. Além disso, a comunicação estabelece o aspecto humano do cuidado e o estreitamento da relação entre equipe e paciente ${ }^{(21)}$.

A explicação do procedimento para diminuir o desconforto e promover cooperação é importante. O paciente pode ser informado sobre o desconforto nasal, náuseas e lacrimejamento que poderá sentir e sobre a forma como poderá cooperar com o procedimento por meio da respiração bucal e deglutição que facilitarão o avanço da sonda. Assim, a comunicação terapêutica necessita estar presente nas interações no cotidiano nas instituições de saúde, a fim de garantir uma assistência integral $^{(21)}$.

De acordo com a recomendação dos juízes, houve alteração na sequência instrucional dos tópicos, ao considerar a obtenção do menor IVC $(0,78)$ e índice Kappa $(0,64)$ no item 2.1 "calça luvas". Dessa forma o item foi modificado para "calça luvas limpas", para garantir a proteção da exposição aos fluidos do paciente e suficiência no uso de luvas limpas não-estéreis ${ }^{(18)}$.

Dessa forma, o item 2.1 "calça luvas" passou a compor o item 2.5 e o item 2.2 "coloca o paciente em 
posição de Fowler" foi transferido para o item 2.1, de acordo com sugestão dos juízes.

Ao item 2.3 "examina a sonda" foi recomendado maior detalhamento, além da supressão do item por já estar contemplado no item 1.3 "seleciona o material e os instrumentos necessários". Desse modo, o item foi excluído do instrumento, pois o exame da estrutura da sonda deve ocorrer durante a seleção dos materiais quando é realizada a busca de defeitos, bordas irregulares e ásperas ou lúmens parcialmente fechados, bem como a validade é observada ${ }^{(17)}$.

Quanto ao item 2.5 "examina as fossas nasais e seleciona a mais permeável" foi sugerido a inclusão de aspectos sobre calibre da sonda. No entanto, essa sugestão não foi aceita, uma vez que o calibre da sonda deve ser observado quando o material é selecionado para a execução do procedimento de acordo com a prescrição médica ${ }^{(17)}$.

No item 2.6 "determina o comprimento da sonda a ser introduzido pela medição NOX", foi sugerido observar na literatura aspectos sobre essa nomenclatura. De acordo com a literatura mais atual ${ }^{(2)}$ a medida NEX vem do inglês para indicar a medição da marca adequada para inserção da sonda, e significa que começa do comprimento do nariz - nose - ao lóbulo da orelha earlobe - até o apêndice xifóide - xiphoid. Logo, o item 2.6 foi alterado para "determina o comprimento da sonda a ser introduzido pela medição NEX".

A reformulação da redação do item 2.7 "marca a medida da distância determinada" foi sugerida. Assim, o item foi redigido da seguinte forma: "marca a sonda no comprimento determinado anteriormente".

Ao item 2.8 "posiciona o paciente" foi sugerido deixar claro que se trata do posicionamento da cabeça do paciente, apesar de alguns juízes afirmarem que o item 2.8 foi contemplado no item 2.2 "coloca o paciente em posição de Fowler" tal sugestão não foi aceita, uma vez que o "posicionamento do paciente" corresponde a flexionar o pescoço do paciente de forma que o queixo se aproxime do tórax, caso não haja contraindicação, a fim de estreitar a traqueia e abrir o esôfago para desse modo favorecer a inserção correta da sonda nasogástrica ${ }^{(22)}$.

Portanto, não corresponde à posição de Fowler, conhecida como Fowler alta, quando se eleva a cabeceira do leito a $45^{\circ}$, caso também não haja contraindicação.

Alguns juízes também afirmaram que o item 2.8 foi contemplado no item 2.11 "introduz a sonda delicadamente na narina". Tal afirmação foi aceita, visto que antes de introduzir a sonda na narina, a cabeça deve ser inclinada para trás para a introdução até a nasofaringe posterior. Esse procedimento ocorre com direcionamento da sonda para baixo e para trás no sentido da orelha e somente quando a sonda alcança a faringe, o paciente deve inclinar a cabeça para frente, pois oclui a via aérea e, desse modo, apresenta menor possibilidade de entrar na traqueia ${ }^{(22)}$. Logo, o item 2.8 foi excluído e o item 2.11 foi alterado para "introduz a sonda delicadamente na narina posicionando a cabeça do paciente".

O item 2.9 "lubrifica a sonda com anestésico" e 2.10 “lubrifica a narina do paciente com anestésico" sofreram permuta de acordo com a sugestão dos juízes. Em relação específica ao item 2.10 "Iubrifica a narina do paciente com anestésico", o texto foi substituído por "utiliza no paciente analgésico tópico", em razão de que a inalação de lidocaína por meio de nebulização, spray e respiração intermitente com pressão positiva por nebulização pela boca, tem demonstrado redução significativa da dor associada à instalação de sonda nasogástrica, apesar destas técnicas exigirem dispositivos especiais nem sempre disponíveis, além de preparações demoradas ${ }^{(19)}$.

Observou-se também em um estudo que a instilação de $5 \mathrm{ml}$ de lidocaína gel $2 \%$, na narina do paciente, cinco minutos antes do procedimento, promove redução significante da dor durante o procedimento de inserção da sonda nasogátrica ${ }^{(19)}$.

No item 2.12 "em caso de resistência, para e retrocede", os juízes afirmaram a necessidade de tornar mais claro o fato de que após retroceder a sonda, há reintrodução desta. A reintrodução pode ocorrer na 
mesma narina ou caso persista, a sonda é retirada, com inserção na outra narina mediante condições propícias para tal ${ }^{(23)}$. Nesse contexto, a redação do item 2.12 ficou "em caso de resistência, para, retrocede e reintroduz a sonda". A mesma orientação de reformulação da redação ocorreu no item 2.14 "observa sinais de tosse, dificuldade respiratória, cianose ou agitação, retirando e reintroduzindo". Assim, para discorrer sobre o fato da interrupção do procedimento mediante sinais de sofrimento ${ }^{(23)}$, o texto foi reescrito dessa maneira: “ao observar sinais de tosse, dificuldade respiratória, cianose ou agitação, retira e reintroduz a sonda"

O último item considerado adequado com alterações foi o 2.16 "confirma a localização da sonda com testes de posicionamentos", que apresentou sugestão para o detalhamento dos testes de posicionamento.

Apesar da técnica de ausculta, ao ser instilado $10 \mathrm{ml}$ ou mais de ar na sonda a fim de observar o ruído na região epigástrica, ser largamente utilizada pelos profissionais para certificação do posicionamento, não há nenhuma evidência que sustenta o método de ausculta para confirmação do posicionamento correto da sonda ${ }^{(16)}$.

Nesse contexto, o teste do hidrogênio $(\mathrm{pH})$ e verificação por raio-X, são considerados testes mais confiáveis pela evidência científica ${ }^{(15-16)}$. A verificação do comprimento exterior da sonda, volume do aspirado, bem como a descrição do aspirado apresentam moderado grau de confiabilidade para avaliação da localização na região gástrica ou jejunal de acordo com a avaliação da evidência ${ }^{(15)}$.

Em relação à avaliação da localização gástrica ou endotraqueal, são considerados no teste do hidrogênio, que os valores de $\mathrm{pH}$ menores que 5 do aspirado apresentam $90 \%$ de chance de indicar o correto posicionamento da sonda no ambiente gástrico ou $77 \%$ de chance, se houver uso de inibidor de ácido ${ }^{(24)}$. Ao passo que, o valor mínimo de $\mathrm{pH}$ endotraqueal é de $6^{(24)}$. Desse modo, para níveis de $\mathrm{pH}$ maiores que 5 é adequado utilizar a radiografia de tórax, padrão ouro, para garantir o correto posicionamento da sonda ${ }^{(24)}$.
Quanto ao aspecto do conteúdo gástrico ou endotraqueal, estudo de Gilbertson ${ }^{(24)}$ apontou não haver distinção de coloração entre os dois aspirados, uma vez que as cores mais observadas foram percebidas nos dois tipos de aspirados.

Os itens que corresponderam às etapas finais da técnica obtiveram índice de concordância perfeito, bem como os itens pertencentes ao bloco de observações finais.

Nesse contexto, torna-se fundamental o registro do procedimento executado por enfermeiros ${ }^{(14)}$. Apesar de estudos $^{(25)}$ apontarem inadequações nessa prática, o registro claro e preciso das informações relacionadas à inserção, administração e à evolução do usuário se constitui atividade indispensável à execução dessa técnica de enfermagem ${ }^{(14)}$.

\section{CONCLUSÃO}

O instrumento da habilidade técnica de sondagem nasogástrica apresentou, por meio da avaliação dos juízes, confiabilidade e fidedignidade para observação da qualidade da assistência de enfermagem, entretanto, alguns itens necessitaram de alterações.

A técnica de sondagem nasogástrica é um procedimento realizado na rotina dos serviços hospitalares que, ultimamente, tem se estendido para atenção domiciliar com os novos programas de internação em domicílio. A sondagem nasogástrica é uma prática fundamental na assistência de enfermagem que exige habilidade e conhecimento.

Portanto, este estudo representa uma etapa fundamental no início do processo de validação do instrumento de habilidade de sondagem nasogástrica.

Os instrumentos válidos quanto ao seu conteúdo são ferramentas adequadas para observação da qualidade da assistência de enfermagem e podem ser aplicados por serviços de saúde para melhoria na capacidade técnica da equipe de enfermagem, bem como em instituições formadoras para observar a habilidade dos alunos de 
graduação, em laboratórios de habilidades ou em campos de prática.

As limitações do estudo correspondem à técnica de seleção de juízes, que impossibilitou a inclusão de juízes de diferentes regiões do país, bem como à necessidade da realização de outras fases desta pesquisa, pois este estudo se propôs a cumprir algumas fases do processo de validação e necessita da ressubmissão dos instrumentos

\section{REFERÊNCIAS}

1. Marshall AP, Cahill NE, Gramlich L, MacDonald G, Alberda C, Heyland DK. Optimizing nutrition in intensive care units: empowering critical care nurses to be effective agents of change. Am J Crit Care [internet]. 2012 [acesso em: 22 fev 2015]; 21(2):186-194. Disponível em: http://ajcc.aacnjournals.org/content/21/3/186.short

2. Malta MA, Carvalho Junior AF, Andreollo NA, Freitas MIP. Medidas antropométricas na introdução da sonda nasogástrica para nutrição enteral empregando a esofagogastroduodenoscopia. Arq Bras Cir Dig [internet]. 2013 [acesso em: 20 fev 2015]; 26(2):107-111. Disponível em: http://www.scielo.br/scielo.php?pid=S0102-

\section{$67202013000200007 \&$ script $=$ sci_arttext\&tlng=pt}

3. Gomes Júnior CAR, Lustosa SAS, Matos D, Andriolo RB, Waisberg DR, Waisberg J. Percutaneous endoscopic gastrostonomy versus nasogastric tube feeding for adults with swallowing disturbances. The Cochrane Library [internet]. 2012 [acesso em: 19 fev 2015]. 1-50.

Disponível em: http://www.update-

software.com/bcp/wileypdf/en/cd008096.pdf

4. Yardley IE, Donaldson L. Patient safety matters: reducing the risks of nasogastric tubes. Clinical Medicine [internet]. 2010 [acesso em: $22 \mathrm{fev}$ 2015]; 10(3):228-230. Disponível em:

http://www.clinmed.rcpjournal.org/content/10/3/228.full.pdf+html

5. Agha R, Siddiqui MRS. Pneumothorax after nasogastric tube insertion. J R Soc Med Sh Rep [internet]. 2011 [acesso em: $24 \mathrm{fev}$ 2015]; 28(2):1-4. Disponível em:

http://shr.sagepub.com/content/2/4/28.full.pdf+html

6. Ribeiro MCB, Lopes LR, Souza-Neto JC, Andreollo NA. Estenose esofágica por uso de sonda nasogástrica - reflexão sobre o uso indiscriminado. Arq Bras Cir Dig [internet]. 2011 [acesso em: $22 \mathrm{fev}$ 2015]; 24(3):191-194. Disponível em:

http://www.scielo.br/pdf/abcd/v24n3/a02v24n3.pdf

7. Brugnolli A, Ambrosi E, Canzan F, Saiani L. Securing of naso-gastric tubes in adult patients: a review. Int J Nurs Stud [internet]. 2014 [acesso em: 19 fev 2015]; 51(1): 943-950. Disponível em: http://ac.els-cdn.com/S0020748913003702/1-s2.0S0020748913003702-main.pdf?_tid=c81f0508-ba0b-11e4-be3800000aab0f27\&acdnat=1424552229_54d948f3bad605821f2097c6a7ea $\underline{81 \mathrm{c} 6}$

8. Vituri DW, Matsuda LM. Validação de conteúdo de indicadores de qualidade para avaliação do cuidado de enfermagem. Rev. esc. enferm. USP [Internet]. 2009 [acesso em: 18 ago 2013]; 43(2): 429:37.

Disponível em: http://www.scielo.br/pdf/reeusp/v43n2/a24v43n2.pdf 9. Bittencourt HR, Creutzberg M, Rodrigues ACM, Casartelli AO, Freitas ALS. Desenvolvimento e validação de um instrumento para avaliação de disciplinas na educação superior. Est. Aval. Educ. [Internet]. 2011 [acesso em: 15 jul 2013]; 22(48):91-114. Disponível em: http://educa.fcc.org.br/pdf/eae/v22n48/v22n48a06.pdf aos juízes para reteste, além da sua aplicação clínica, em busca de indicadores mais precisos e específicos.

É evidente a necessidade de mais estudos de validação quanto ao procedimento de sondagem nasogástrica, mediante sua escassez na área de enfermagem, principalmente sobre as técnicas de inserção e confirmação de posicionamento da sonda nasogástrica. O aprimoramento desse conhecimento acarreta em uma assistência mais qualificada.

10. Alexandre NMC, Coluci MZO. Validade de conteúdo nos processos de construção e adaptação de instrumentos de medidas. Ciênc. saúde coletiva [Internet]. 2011 [acesso em: 01ago 2013]; 16(7): 3061-8. Disponível em: http://www.scielo.br/pdf/csc/v16n7/06.pdf 11. Pasquali L. Instrumentação psicológica: fundamentos e práticas. 1st ed. Porto Alegre: Artmed, 2010.

12. Leães DM, Mello E, Beghetto M, Assis MCS. Enteral feeding tubes: are insertion techniques and positioning based on anatomical evidence? Nutrition and Health [internet]. 2012 [acesso em: $21 \mathrm{fev}$ 2015]; 21(3):193-200. Disponível em:

http://nah.sagepub.com/content/early/2012/09/23/02601060124599 37.full.pdf+html

13. Resolução № 63 da Agência Nacional de Vigilância Sanitária, de 6 de julho de 2000 (BR). Aprova o Regulamento Técnico para fixar os requisitos mínimos exigidos para a Terapia de Nutrição Enteral. Diário Oficial da União. 6 jul 2000.

14. Resolução $N^{\circ} 277$ do Conselho Federal de Enfermagem, de 16 de junho de 2003 (BR) [Internet]. Aprova as normas de procedimentos a serem utilizadas pela equipe de Enfermagem na Terapia Nutricional. Diário Oficial da União. 16 jun 2003 [acesso em: 17 abr 2013]. Disponível em: http://novo.portalcofen.gov.br/resoluo-cofen2772003_4313.html

15. Stepter CR. Maintaining placement of temporary enteral feeding tubes in adults: acritical appraisal of the evidence. Med Surg Nurs[Internet]. 2012 [acesso em: 07 maio 2013]; 21 (2): 61-9. Disponível em: http://www.ncbi.nlm.nih.gov/pubmed/22666997 16. Tho PC, Mordiffi S, Ang E, Chen H.Implementation of the evidence review on best practice for confirming the correct placement of nasogastric tube in patients in an acute care hospital.Int J Evid Based Health [Internet]. 2011 [acesso em: 02 maio 2013]; 9: 51-60. Disponível em: http://onlinelibrary.wiley.com/doi/10.1111/j.17441609.2010.00200.x/full

17. Ciosak SI, Matsuba CST, Silva MLT, Serpa LF, Poltronieri MJ. Acessos para terapia de nutrição parenteral e enteral. Sociedade Brasileira de Nutrição Parenteral e Enteral e Associação Brasileira de Nutrologia [internet]. 2011 [acesso em 25 fev 2015]; 2-10. Disponível em: http://www.projetodiretrizes.org.br/9_volume/acessos_para_terapia_ de nutricao parenteral e enteral.pdf

18. Ferreira AM, Bertolo D, Andrade MR, Andrade D. Conhecimento da equipe de enfermagem acerca do uso de luvas no contexto hospitalar. Rev Eletr Enf [Internet]. 2009 [acesso em 19 fev 2015]; 11(3):628-34. Disponível em: http://www.fen.ufg.br/revista/v11/n3/v11n3a21.htm. 19. Uri O, Yosefov L, Haim A, Behrbalk E, Halpern P. Lidocaine gel as an anesthetic protocol for nasogastric tube insertion in the ED. Am J Emerg Med [internet]. 2011 [acesso em: 25 fev 2015]; 29:386-390. Disponível em: 
http://www.sciencedirect.com/science/article/pii/S073567570900535 X

20. Lin HJ, Foo NP, Guo HR, Lin CC, Lin CC, Chen KT. Assessment of a new method to verify feeding tube placement by syringe aspiration in a porcine model. Int J Gerontol [internet]. 2012 [acesso em: $25 \mathrm{fev}$ 2015]; 6:220-223. Disponível em:

http://www.sciencedirect.com/science/article/pii/S187395981200041 $\underline{5}$

21. Negreiros PL, Fernandes MO, Costa KNFM, Silva GRF. Comunicação terapêutica entre enfermeiros e pacientes de uma unidade hospitalar. Rev Eletr Enf [internet]. 2010 [acesso em 18 fev 2015]; 12(1):120-132. Disponível em:

http://www.revistas.ufg.br/index.php/fen/article/view/9529/6598

22. Kirtania J, Ghose T, Garai D, Ray S. Esophageal guidewire-assisted nasogastric tube insertion in anesthetized and intubated patients: a prospective randomized controlled study. Anesth Analg [internet]. 2012 [acesso em: 25 fev 2015]; 114(2):343-348. Disponível em: http://journals.Iww.com/anesthesia-

analgesia/Abstract/2012/02000/Esophageal_Guidewire Assisted Naso gastric_Tube.15.aspx

23. Chun DH, Kim NY, Shin YS, Kim SH. A randomized, clinical, trial of frozen versus standard nasogastric tube placement. World J Surg [internet]. 2009 [acesso em 24 fev 2015]; 33(1):1789-1792. Disponível em: http://link.springer.com/article/10.1007/s00268-009-0144-x 24. Gilbertson HR, Rogers EJ, Ukoumunne OC. Determination of a pratical $\mathrm{pH}$ cutoff level for reliable confirmation of nasogastric tube placement. JPEN J Parenter Enteral Nutr [internet]. 2011 [acesso em: 26 fev 2015]; 35(4):540-544. Disponível em:

http://pen.sagepub.com/content/35/4/540.short.

25. Silva JA, Grossi ACM, Haddad MCL, Marcon SS. Avaliação da qualidade das anotações de enfermagem em unidade semi-intensiva. Esc Anna Nery [internet]. 2012 [acesso em 26 fev 2015]; 16(3):576-581. Disponível em: http://www.scielo.br/pdf/ean/v16n3/21.pdf.

Recebido: 10/03/2014.

Aceito: 04/02/2015.

Publicado: 30/06/2015. 